\title{
La cartografía social como herramienta de investigación participativa del territorio. Diagnosis de paisajes ancestrales en comunidades indígenas de la Amazonia ecuatoriana.
}

\author{
Paulo Carrión* \\ Universidad Técnica Particular de Loja (Ecuador) \\ María Yolanda Pérez Albert** \\ Universitat Rovira i Virgili (España)
}

\begin{abstract}
Resumen: El análisis del paisaje y su caracterización se realiza habitualmente bajo miradas cuantitativas, es decir, su valor se establece en relación a sus características físicas y a la potencial explotación de sus recursos. Sin embargo, la realización de diagnosis y diagnósticos desacertados y la aplicación de estrategias de gestión poco eficaces, cuestionan estas metodologías y muestran la necesidad de generar nuevas epistemologías y métodos de investigación cualitativos sobre la relación sujeto-lugar. El objetivo es analizar el potencial de la cartografía social como herramienta de investigación-acción participativa (IAP) para representar y caracterizar paisajes ancestrales de la Amazonia ecuatoriana. Para ello se han considerado narrativas comunitarias que permiten registrar tanto elementos físicos del territorio como simbólicos y culturales. Se concluye que la cartografía social es una estrategia fundamental de soberanía e instrumento político para tomar conciencia de la realidad de las comunidades tanto desde su interior como desde el exterior.
\end{abstract}

Palabras Clave: Cartografía social; Comunidades indígenas; Paisaje; Investigación acción participativa; Amazonía.

Social mapping as a tool for participatory research in the territory. Diagnosis of ancestral landscapes in indigenous communities of the Ecuadorian Amazon.

Abstract: The analysis of the landscape and its characterization is usually carried out from quantitative perspectives, that is, its value as established in relation to its physical characteristics and the potential use of its resources. However, misguided diagnoses and the application of ineffective management strategies question these methodologies and reveal the need to generate new epistemologies and qualitative research methods for the subject-place relationship. The objective is to analyze the potential of social cartography as a participatory action research tool (IAP) to represent and characterize ancestral landscapes of the Ecuadorian Amazon. To this effect, the community narratives with respect to place have been used to register elements in their symbolic and cultural values. It is concluded that social cartography is a fundamental strategy of sovereignty and a political instrument to offer a true perspective of the community sense of place from within and without.

Keywords: Social cartography; Indigenous communities; Landscape; Participatory action research; Amazon.

\section{Introducción y objetivos}

La complejidad inherente a la investigación y caracterización del paisaje ha generado muchas controversias en las diferentes ciencias que han intentado analizar e interpretar sus significados; diversas disciplinas se han ocupado de estudiarlo a partir de enfoques contemplativos, reduciendo su estudio a ordenamientos taxonómicos y afincando su importancia en aspectos medibles y estimaciones

* Universidad Técnica Particular de Loja (Ecuador); E-mail: pccarrion47@utpl.edu.ec; https://orcid.org/0000-0002-7515-2557

** Universitat Rovira i Virgili (España); E-mail: myolanda.perez@urv.cat; https://orcid.org/0000-0003-1634-4986 
visibles. Si bien es cierto que esto responde a necesidades concretas, ha configurado una separación ontológica (aparente) entre el ser humano y el entorno, superponiendo la condición del ser humano y colocando la existencia del paisaje en un estado de subordinación del cual la humanidad se sirve, de modo que su valor está en función de la utilidad que se le pudiera dar. Esta idea de ruptura y división comulgada con una concepción materialista de la historia instituye el pilar fundamental de la forma en cómo comprendemos el territorio, cómo nos relacionamos con sus elementos, cómo los representamos y cómo los leemos. Así, en el ámbito de la administración pública se ha pretendido caracterizar el paisaje desde la normativa y no desde el contexto y el consenso (Diez Tetamanti et al., 2012).

Las metodologías utilizadas son estándares y, si bien dentro de racionalidades objetivas y numéricas cumplen con un papel fundamental en la efectividad funcional de su análisis y son la base de las estrategias de su planificación ordenación y actuación, excluyen formas epistemológicas alternativas arraigadas en la teoría social. Esta teoría social aplicada al paisaje permitiría conocerlo e interpretarlo desde su hondura, no solo a través de dimensiones y enfoques objetivistas sino también existencialistas. Uno de los problemas principales que existe en los procesos de desarrollo local es la falta de comprensión del territorio por parte de las instituciones responsables, resultado de las formas en que se construyen los acercamientos y diálogos con la población local; muchas veces estos diálogos se encuentran cargados de intereses particulares y son estereotipados, lo que aumenta la resistencia de las comunidades, reduce y distorsiona las subjetividades eliminando los rasgos singulares y característicos de los territorios (Guatarri, 2000). En virtud de ello es necesario replantear recursos, incorporar métodos de exploración social y actuaciones en escenarios participativos e interactivos, fuera de las lógicas convencionales, que permitan equilibrar las desigualdades entre una investigación tecnocrática y mecánica del territorio y las narrativas y dimensiones de los grupos poblacionales que en él habitan. En este contexto, este artículo tiene como objetivo analizar la potencialidad de la cartografía social como herramienta de investigación-acción participativa (IAP) para representar y caracterizar paisajes ancestrales de la Amazonia ecuatoriana. En primer lugar, se reflexiona sobre diferentes aproximaciones teóricas que permitan poner en contexto la investigación. En segundo lugar, se exponen las características de la IAP, de la cartografía participativa y de la propuesta metodológica diseñada. Posteriormente se presentan los resultados que corresponden a un análisis-diagnóstico de las áreas de intervención a partir de las representaciones resultantes de los talleres de cartografía participativa. Finalmente se expone una valoración del grado de participación y percepción de las comunidades en el estudio.

\section{Rasgos distintivos de la Investigación-acción participativa}

La Investigación-acción participativa (IAP, de aquí en adelante) como modelo de investigación psicosocial se concibe desde un interés emancipatorio (Sirvent y Rigal, 2012), es decir de empoderamiento, e ideológico, y está orientada a la transformación de la realidad desde dentro. Esta concepción implica la participación de la comunidad como autogestora del proceso de investigación, desde la diagnosis hasta la propuesta de mejora. Este enfoque de carácter cualitativo se distingue de otros en la forma en que se aborda el individuo de estudio, sus intenciones y propósitos; no únicamente cumple como una acción investigadora, sino que se investiga en, con y para la comunidad. Esta posición de participación abierta promueve la colaboración de los miembros del colectivo independientemente de su nivel de educación o posición social con miras a generar reflexiones amplias a partir de interacciones dialécticas dentro del conocer, el analizar, el comprender y el proponer. Según Fals Borda (2008) la IAP no solo forma parte de una serie de métodos de experimentación y análisis, sino que se manifiesta como una filosofía de vida (Fals Borda, 2008 en Colmenares, 2012) ya que promueve el desarrollo de la comunidad y estimula el espíritu de cooperación y organización de sus miembros (Balcázar, 2003). De esta manera la IAP es vista como una apuesta onto- epistémica que se resuelve a partir de dos elementos: (1) la experiencia psicológica del sujeto que conoce y (2) el "individuo "conocido. En función de estos dos elementos se establecen dos preguntas claves en el desarrollo de una IAP: (1) ¿Cómo sabe la experiencia psicológica del sujeto que conoce, que el "individuo" conocido existe efectivamente; (2) ¿El individuo conocido, existe tal y como lo concibe la experiencia psicológica? A diferencia de las metodologías convencionales de investigación social en donde el proceso de exploración-intervención se fundamenta en el conocimiento a priori, que condiciona la forma en que comparece el "individuo" conocido frente al sujeto que conoce, en la IAP se incluye la incertidumbre como presupuesto necesario dentro de una construcción colectiva permanente del conocimiento. En tal sentido el "individuo" conocido se presenta desde una condición y estado original. La IAP, por lo tanto, busca trascender las costumbres pedagógicas con el propósito de proyectar al grupo 
poblacional intervenido como protagonista central de sus propias construcciones y valoraciones. Desde una perspectiva ideológica, la IAP se convierte en un instrumento de resistencia frente a imposiciones que van en contra de valores e intereses locales. Desde el punto de vista epistemológico la IAP trasgrede metodologías tradicionales, en donde se manifiestan relaciones de poder ejercidas por el investigador y posicionamientos pasivos por parte de los investigados, y compromete a los involucrados a que dirijan su propio proceso de exploración, lo que en la práctica hace que la investigación fluctúe, de modo que sus momentos son adaptables y configurables de acuerdo a los diferentes contextos.

En resumen, en la IAP no existen vínculos posicionales o jerárquicos entre los investigadores y los investigados de ahí que los actores sociales no son objeto de estudio de los cuales se obtienen información, sino que se convierte en agentes-sujetos activos, con la capacidad de analizar, proponer y planificar (Rocha, 2016).

\section{La cartografía social como herramienta IAP y registro de la memoria territorial indígena}

En relación a la cartografía social, cabe destacar los trabajos pioneros de Guillermo Furlong (1936), las aproximaciones conceptuales de Andrade y Santamaría (2006), las experiencias de mapeo participativo de la United Nation Human Settlementes Programme (2010) o los trabajos con comunidades rurales del Fondo Internacional de Desarrollo Agrícola (2009).

La tarea de intentar caracterizar los territorios a través de metodologías participativas, incluyentes y cooperativas que impulsen los auto-reconocimientos y reflejen las verdaderas necesidades, intereses y saberes de los grupos poblacionales intervenidos se ha planteado desde diferentes enfoques. Dentro de estas metodologías se encuentra la cartografía social como un instrumento multidimensional de conocimiento, ordenamiento y resistencia territorial (Barrera, 2009). La cartografía social ha tenido varias calificaciones expresadas de acuerdo a sus atributos, procesos aplicativos y características metodológicas. Algunos de los términos encontrados para identificarla son: contra cartografía, etno-cartografía, cartografía comunitaria o cartografía indígena. Todos basan su principio en la implicación activa de la población del territorio investigado dentro de sistemas y procesos dialógicos (Fals Borda, 1987) posibilitando la caracterización del territorio en la comunidad; de hecho, se podría decir que el primer objetivo de la cartografía social es el involucramiento y participación de sus actores y el segundo, la reflexión colectiva que motive la capacidad propositiva y permita trazar nuevas rutas que respondan tanto a transformaciones estructurales como subjetivas del territorio. Como recurso y registro testimonial de la memoria histórica, la cartografía social se convierte en un procedimiento, un método, una máquina de creación y recuperación de significados (Diez, Temantine. et al, 2016) muchas veces desapercibidos o desaparecidos pero que han dado como consecuencia sistemas de valores, constructos colectivos y códigos que condicionan cotidianamente las formas en que las comunidades se relacionan con su entorno.

Lo nuclear y significativo dentro del proceso cartográfico social imbrica dos elementos fundamentales; por una parte, la proyección ilustrativa que corresponde a los elementos pictóricos y componentes retóricos que se moldean dentro de la representación como resultado final del ejercicio y, por otra parte y más importante, las intencionalidades e intereses que se desarrollan y exponen a través de las relaciones discursivas de los participantes (Lois, 2009). En este sentido, resulta necesario subrayar la importancia que tiene el discurso dentro de lo estructural, lo funcional y lo simbólico del ejercicio cartográfico. Para Foucault (1988) el discurso como expresión y conjunto de enunciados históricamente instituido es controlado organizado y seleccionado dentro de un sistema-relación de saber y poder. Así los hechos ocurridos en el territorio validado a través de registros o tradiciones orales, no solo son exhibidos dentro de las dinámicas dialógicas entres los participantes y el investigador, sino que muestran en la configuración del discurso (códigos, signos, símbolos) las formas en que las comunidades entienden y se relacionan con el territorio y consigo mismas. Por ello la historicidad no se perfila posterior al ser, sino más bien es la que le da origen; de esta manera el ejercicio cartográfico participativo se convierte no solo en un catálogo de significados, sino en dispositivo "episteme" y en una herramienta didáctica.

Tal y como se ha dicho, en la cartografía social los procesos de aprendizaje constituyen una acción recíproca, superando cualquier tipo de posiciones jerárquicas o de subordinación. Así, el conocimiento se produce a través de un modelo relacional genuino donde el individuo conocido que antes se consideraba un objeto pasivo (que), ahora se transforma en un sujeto activo (quien) difuminando las fronteras o dicotomías que pudieran existir entre el investigador o sujeto que conoce y el investigado. En otras palabras, el investigador se implica con el sujeto conocido dentro de un sistema de acuerdos despolarizados que incluyen coproducción y convergencia (Kastrup, 2014). Estas experiencias comunes generadas a 
partir relaciones horizontales se conciben por medio de sinergias positivas que ponen de manifiesto valores como la equidad, la solidaridad y reciprocidad pro un objetivo común. Similar al carácter del ejercicio cartográfico social, encontramos prácticas ancestrales y tradiciones de trabajo comunitario como la minga, o minka en kichwa que se manifiesta como un sistema-red de apoyo que implica el compromiso y el esfuerzo colectivo por un interés comunal. La cartografía social, al igual que la minka, es un proceso participativo que permite a los grupos poblacionales fomentar formas de organización y relaciones de interdependencia que influyen significativamente sobre la realidad a la que pertenecen.

\section{4. Áreas de intervención, contexto y justificación}

El Parque Nacional Yasuní (PNY) se crea en 1979 con el objetivo de proteger el bosque húmedo tropical (Villaverde, 2005) (Figura 1); en el año 1989 una extensión de más de 2 millones de ha, fue incorporada a la Red Mundial de Reservas de la Biósfera (UNESCO); en 1999, una porción de aproximadamente 700.000 hectáreas fue declarada como "Zona Intangible" (ZI) con el fin de salvaguardar a los pueblos indígenas en aislamiento voluntario. Calificado como refugio del pleistoceno, el Parque Nacional Yasuní se convierte en Patrimonio Natural de la Humanidad y en una de las áreas protegidas más valoradas e importantes del Ecuador albergando 2500 especies de árboles, 300 especies de mamíferos, 150 especies de anfibios, 121 especies de reptiles, 559 especies de aves (Bass, et al. 2010). A pesar de su significado natural y cultural, un alto porcentaje del PNY se ha concesionado a empresas mineras generando graves impactos ambientales y sociales. La pérdida de hábitats ha tenido como consecuencia que especies de flora y fauna se encuentren en peligro de extinción, algunas de ellas aparecen en las "listas rojas" del Ecuador y en las "listas urgentes" de la International Union for Conservation of Nature- UICN. Los procesos de extracción de crudo en la Amazonia se inician en 1970 y continúan en una segunda etapa de expansión con la construcción del oleoducto de crudos pesados en el año 2001. A día de hoy, la explotación de crudo no solo no ha parado, sino que ha entrado en un periodo de bonanza y apogeo, configurándola como el principal sector de "dinamización económica" y convirtiendo al Ecuador en un país dependiente del petróleo.

En 2007 el Gobierno Ecuatoriano lanza una propuesta denominada YASUNI ITT, derivada de la necesidad de resolver la dicotomía entre conservar la Reserva de la Biosfera Yasuní, como el mayor patrimonio natural que tiene el país, o explotar parte de los campos petroleros que se encuentran en este lugar. La propuesta planteaba a la Comunidad Internacional la posibilidad de evitar la emanación de 407 millones de toneladas de dióxido de carbono producto de la posible explotación de los campos, a cambio de que ésta, la Comunidad Internacional, se comprometiera a solidarizarse con el 50\% de los ingresos que potencialmente se podrían obtener de los yacimientos de petróleo. La estrategia tenía una duración de 14 años y dentro de los acuerdos de negociación se preveían dos escenarios: el primero (positivo), la no explotación si al termino de 7 años se obtenía al menos el $40 \%$ de la mitad del monto esperado y el segundo escenario (obligado), la explotación a causa del fracaso del primero (Becerra, 2016). En 2014 se evaluó lo recaudado dando como resultado que el monto obtenido no superó el 0.05\% de lo previsto, por lo que el Presidente de la Republica de aquel periodo, Rafael Correa Delgado, toma la decisión de iniciar los procesos de exploración y explotación en la Reserva. La propuesta YASUNI ITT se presentaba como una alternativa post petrolera frente a un modelo extractivo y una estrategia de conservación del mayor patrimonio natural de los ecuatorianos, de los territorios ancestrales y de las culturas milenarias que habitan la zona (Vallejo, 2012) pero no tuvo el seguimiento esperado.

En febrero de 2018 se realiza en Ecuador una Consulta Popular en la cual se pregunta a la ciudadanía - se cita literalmente - ¿Está de acuerdo con incrementar la zona intangible en al menos 50.000 hectáreas y reducir el área de explotación petrolera autorizada por la Asamblea Nacional en el Parque Nacional Yasuní de 10.03 hectáreas a 300 hectáreas? A pesar de haber ganado el SÍ, las interpretaciones son difusas; en la segunda parte de la pregunta se trata de una disminución del área de explotación en el parque asumiéndose que el problema principal de la actividad extractiva es la cantidad de territorio, omitiendo cuestiones fundamentales de impacto que muchas veces son independientes a la dimensión del espacio explotado. Así mismo, tampoco se menciona en dónde se va a realizar la reducción del área petrolera ni qué criterios se van a utilizar para identificar la zona. Estos y algunos otros interrogantes muestran la ambigüedad de los planteamientos y las dudas que se generan alrededor del futuro de uno de los lugares más biodiversos del mundo. Es incuestionable que la gestión y conservación del parque ha estado en el centro de muchos debates y controversias; sin embargo, a pesar del interés que ha ocasionado, las actividades extractivas siguen constituyendo la base de un conflicto social, económico, 
cultural, político y ambiental. Las estrategias generadas en torno a su conservación han sido insuficientes, tanto cualitativamente como cuantitativamente, y excluyentes en relación a la opinión de las comunidades indígenas cuyos conocimientos ancestrales están relacionados con los sistemas naturales del parque y su equilibrio ecológico.

Estos antecedentes justifican la necesidad de una IAP, que se acerque a los territorios en conflicto a través de una mirada contextual-relacional y sistémica, que genere diagnósticos y propuestas participativas, y que ponga en valor los conocimientos, percepciones, impresiones y sentires de las comunidades indígenas. Las áreas de intervención en la consecuente IAP corresponden a una sección oriental del río Napo y del río Tiputíni dentro del Parque Nacional y Reserva de la Biosfera Yasuní, ubicada ésta en la región amazónica del Ecuador. Se han seleccionado tres comunidades indígenas de nacionalidad kichwa: la comunidad Chiro Isla, la comunidad Alta Florencia y la comunidad Llanchama (Figura 1).

\section{Figura 1: Ubicación geográfica de los territorios de intervención,} delimitación de los bloques petroleros y ubicación de los pozos.

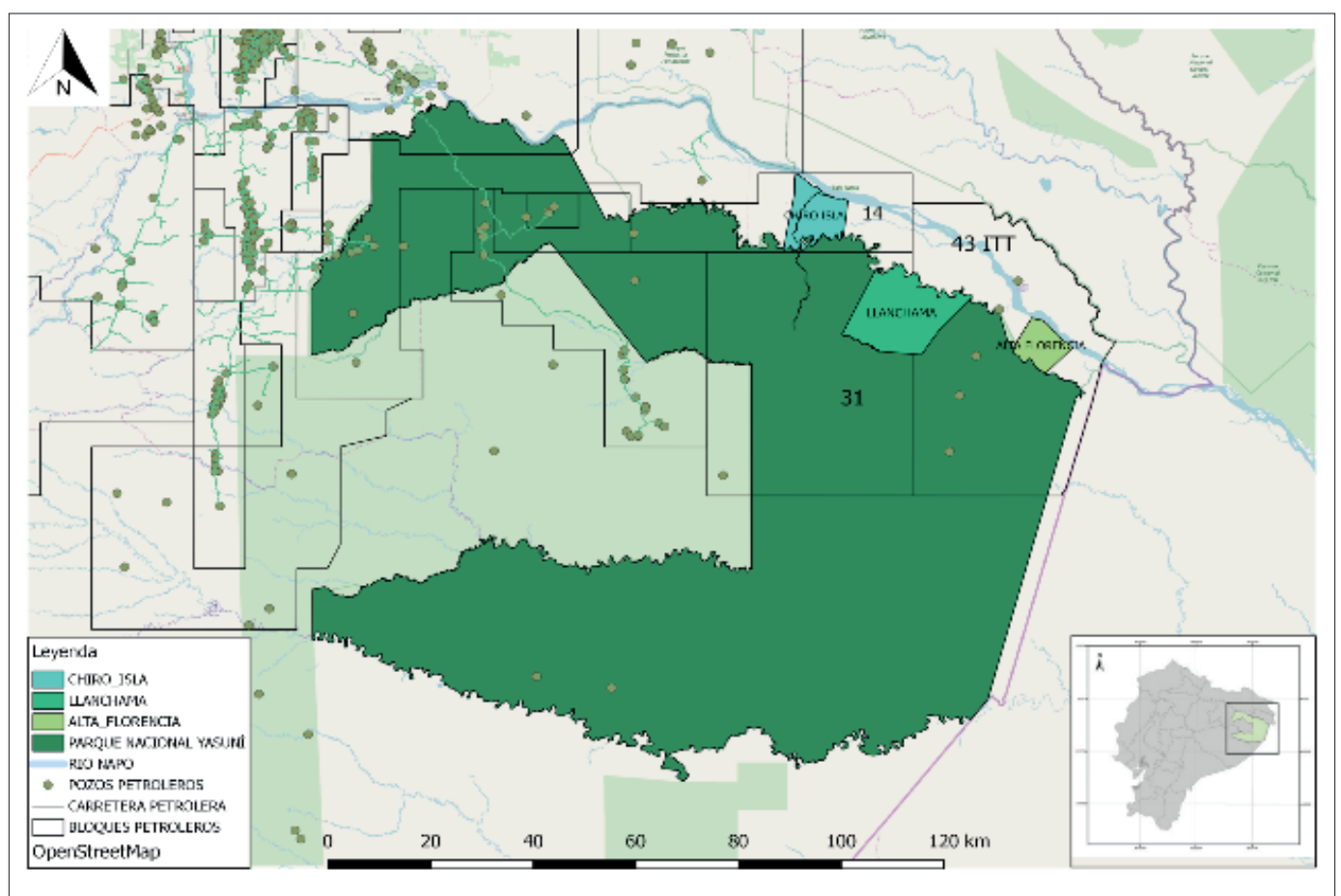

Fuentes: Ministerio del Ambiente/Ecuador, Secretaria de Hidrocarburos del Ecuador. Cartografía base: Raster- OpenStretMap.

Elaboración propia.

\section{Consideraciones metodológicas}

La IAP, al igual que la investigación científica, está orientada a la construcción de un objeto científico, pero de naturaleza subjetiva y de energía colectiva (Rigal y Sirvent, 2011; Sirvent, Rigal, 2012). La energía colectiva manifestada en el trabajo en equipo constituye el eje vertebrador del proceso investigativo participativo. En este sentido es necesario diferenciar ciertas cualidades que caracterizan y diferencian a la IAP de otras metodologías de investigación. Para Rocha (2016) existen algunos elementos a tomar en cuenta para considerar una investigación como un proceso activo y participativo: (1) la subjetivación del individuo de intervención; esto suprime las asimetrías del conocimiento y las relaciones de poder, 
poniendo en equilibrio los intereses, necesidades y saberes del investigador externo y los investigados (investigadores internos). (2) Las demandas de los sujetos; estas serán consideradas y tomadas en cuenta como aspectos fundamentales y prioritarios en el diseño y planteamiento de la hoja de ruta a ejecutar dentro del proceso exploratorio. (3) Unión de la reflexión a la acción; esto involucra el hecho que los participantes dentro del sentido praxiológico de la IAP, desarrollan una conciencia crítica a medida en que sucede su participación. (4) Comprensión de la realidad como una complejidad; presupone la necesidad de entender que la realidad se constituye a partir de aspectos ciertos e inciertos, en permanente mutación y cambio, por lo cual las estrategias de intervención dentro del proceso exploratorio serán configurables de acuerdo a los contextos y sus complejidades. (5) Construcción de creatividades colectivas; significa entender que las significaciones comunes se crean dentro de sinergias e inter-aprendizajes. (6) Comunicación dialógica como discurso colaborativo y cooperativo; en la IAP la comunicación entre los participantes se caracteriza por su interacción directa. Este tipo de comunicación al ser espontánea y no rigiéndose por ningún tipo de reglas estructuradas, elimina jerarquías, expande los niveles de confianza y otorga libertad para los saberes. (7) Construcción de reflexividades colectivas; la reflexividad colectiva es la capacidad de los sujetos para comprender, construir y deconstruir su propia realidad, se convierte por lo tanto en el pilar fundamental del intercambio de reflexiones y constituye el inicio y el final de un proceso de investigación participativa. Como resumen podemos decir que en la IAP se organizan dos ejes, por una parte, el eje organizativo que involucra la planificación y la observación y el estratégico que comprende la acción y la reflexión (Kemmis 1998, en Colmenares, 2012).

En virtud de lo expuesto y haciendo caso al carácter flexible que caracteriza a la IAP, esta investigación presenta cuatro fases que participan sistemática y consecutivamente en el proceso de investigación. I Hallazgo; II. Diseño de la hoja de ruta de intervención; III. Intervención; y IV. Valoración.

\section{Fase I. Hallazgo}

Corresponde a las consideraciones teóricas e hipótesis iniciales en base a la búsqueda, recolección y clasificación de la información relacionada a la problemática planteada. En esta etapa inicial de la exploración se lleva a cabo la indagación de testimonios a través de diálogos abiertos y entrevistas semiestructuradas con miembros de entes gubernamentales y privados para definir los objetivos de cada una de las etapas de la fase de intervención y diseñar la hoja de ruta. Así mismo se recopila información documental y estadística que servirá para tener una idea-antecedente de la configuración territorial de las áreas de intervención (sociedad, economía, medio ambiente, actividades extractivas, dinámicas sociales y cultura), de acuerdo a esto se pretende que la información consolidada provenga tanto de fuentes primarias como secundarias, internas como externas, desde entornos físicos hasta virtuales.

\section{Fase II. Diseño de la hoja de Ruta}

Implica la propuesta de planificación dentro de las actividades a realizar. Una vez que se han definido participativamente los objetivos del proceso de exploración y se haya respondido a la interrogante de que es lo que se quiere lograr, se organiza con estructura y método las tareas a ejecutar; la hoja de ruta buscará responder dos preguntas fundamentales ¿Cómo se lograrán los objetivos propuestos? y ¿Cuándo se alcanzarán los mencionados objetivos? Para que esta herramienta sea útil se intentarán separar las actividades en función de dos contenidos estratégicos.

- Contenido 1. Contacto Inicial: es el primer acercamiento con el territorio y los problemas a investigar. Las tareas se inician con encuentros y diálogos con los principales agentes comunitarios involucrados para establecer acuerdos y compromisos frente a aspectos de logística y voluntades.

- Contenido 2. Socialización del proyecto: como proceso de interacción, la socialización del proyecto pretende, por un lado, dar a conocer a la comunidad los componentes, objetivos, propósitos y actividades a desarrollar en el proceso exploratorio y, por otro, generar mecanismos bidireccionales de interiorización, que permitan ajustar las acciones consecuentes de la investigación a las percepciones locales. En esta etapa se define el cronograma de actuación que se desarrollará dentro de la fase de intervención, se establecen las funciones de cada uno de los miembros del equipo de investigación y responsables comunitarios, se fija el lugar en donde se desarrollarán los talleres cartográficos considerando aspectos de facilidad y proximidad con el mayor número de familias y se establecen compromisos para el desarrollo óptimo del ejercicio. 


\section{Fase III. Intervención.}

La intervención corresponde a la ejecución del ejercicio cartográfico. Dentro de la estructura metodológica de esta fase se plantean algunas etapas en concordancia con la propuesta del Fondo Internacional de Desarrollo Agrícola (2010).

- Adecuación: El proceso de adecuación se inicia haciendo una presentación a la comunidad del proyecto, se expone sobre los agentes que intervienen, los objetivos que se pretenden alcanzar; se proporciona a la comunidad información general sobre el ejercicio cartográfico, el por qué y para qué de la actividad, los posibles conflictos que se podrían encontrar en su ejecución, la importancia que tiene la participación comunitaria en dicho proceso y la potencialidad que tendrían los resultados obtenidos. Una vez que se ha establecido un escenario de confianza entre los miembros de la comunidad y el equipo investigador, se induce una serie de cuestionamientos que permitan determinar ciertas características propias de la problemática: ¿Cuál es la historia de nuestra comunidad?, ¿Cuáles son los principales conflictos en el territorio?, ¿Cómo se ha venido desarrollando el turismo comunitario en la zona?, ¿Cuáles son nuestras debilidades, amenazas, potencialidades y oportunidades como comunidad?, ¿Por qué es importante revalorizar nuestros territorios-paisajes?, ¿Cuál es la expectativa que se tiene del ejercicio cartográfico?, ¿Cómo la elaboración de mapas nos permitiría repotenciar nuestros paisajes y territorios? Estos cuestionamientos se responden a través de un proceso comunicativo no jerárquico y sinérgico también llamado diálogo de saberes.

- Levantamiento: para realizar un diagnóstico participativo adecuado de acuerdo a las problemáticas e intereses de las comunidades, es necesario abordar el análisis del territorio dentro de una línea temporal que permita realizar estimaciones comparativas. Para ello se proponen dos dimensiones temporales a representar cartográficamente, pasado y presente. La cartografía descriptiva del pasado como ejercicio de reconstrucción de la memoria histórica se situará en un periodo de 30 años atrás e identificará: límites, distribución espacial de la población, principales recursos naturales, atractivos turísticos, problemas y /o conflicto territoriales, medio ambientales e institucionales y elementos del paisaje que se consideren sagrados, que tengan un criterio espiritual o que representen relaciones o conocimientos ancestrales significativos para la comunidad.

El mapa del presente corresponderá a una representación que contenga los mismos elementos que el mapa del pasado, pero en el momento actual; esto servirá para conocer los cambios que el paisaje pudo haber sufrido en el tiempo y extraer conclusiones hipotéticas de las causas de dichas transformaciones. Como complemento de análisis se procedió, dentro del trabajo de campo, a realizar recorridos guiados por miembros de las comunidades para la toma de coordenadas geográficas de senderos y elementos de importancia mayor del paisaje.

En cuanto a la dinámica de la cartografía participativa, se contó con un número mínimo de 15 participantes por comunidad que se distribuyeron en 3 mesas de trabajo de acuerdo a las dimensiones temporales a representar cartográficamente (omitiendo cualquier tipo de selección por sexo, género, edad etc.). Se consultó abiertamente al grupo universo con qué mesa se siente identificado y se divide el conjunto de los participantes en tres grupos; una vez conformados se llevan a cabo reuniones periódicas con cada uno para estimular la participación a través de la introducción de preguntas e indicaciones, de manera que el ejercicio cartográfico se enfoque y el tiempo estimado no se difiera en cuestiones poco trascendentes. Los pasos dentro de la representación cartográfica se resumen de la siguiente manera:

a) Dibujo de Croquis: sin valerse de precisiones, los participantes plasman una idea básica de acuerdo a la imagen mental que tienen del territorio y que refleje de alguna manera su realidad. A través de la inducción de preguntas por parte del equipo de investigación se intentará esbozar la forma del territorio, su delimitación y algunos elementos de caracterización general.

b) Elementos del paisaje / representaciones pictóricas: como se había mencionado anteriormente, lo fundamental dentro de la representación cartográfica no solamente son las imágenes visuales de los elementos que componen el territorio y que pudieran graficarse en el bosquejo general, sino los relatos que se cuentan alrededor de ellas. Las significaciones relacionadas a eventos, deseos y sensibilidades comulgarán en una relación imagen mental-discurso, para darles formas visibles a los elementos que componen el medio físico. Con el objetivo de que la representación tenga un carácter eminentemente endógeno de acuerdo a la articulación de la memoria visual con lo simbólico, los trazos, colores, proporciones y demás, serán elección propia de los participantes 
quedando al margen cualquier tipo de intervención por parte del equipo de investigación que pudiera interferir e influenciar en el resultado final.

c) Interpretación: se abre un espacio de socialización, debate y aportes deliberativos de los mapas obtenidos con el objetivo de validar, aprobar o rechazar la información generada. En esta etapa del ejercicio cartográfico, los participantes deberán observar los mapas para generar sugerencias y reflexiones en torno a los contenidos resultantes. Para ello se tendrán en cuenta algunos elementos: a) Cada grupo designará un relator que se encargará de exponer y explicar ordenadamente el trabajo desarrollado por el grupo, las aportaciones realizadas por los participantes, las ideas principales, inquietudes y dificultades que se encontraron durante el ejercicio y, finalmente, socializará las proposiciones finales de acuerdo a reflexiones grupales. b) Se instaurará y documentará un periodo de discusión con todos los participantes en relación a las polémicas encontradas en las exposiciones; este debate estará moderado por el equipo de investigación con el propósito de que el intercambio de opiniones no arribe a cuestiones que no correspondan al objetivo de la práctica. c) Se realizará un análisis participativo de la información resultante y sistematizada por el equipo de intervención, delimitando su abordaje en antecedentes y consecuencias.

\section{Fase IV. Valoración.}

Corresponde a una lectura crítica de la cartografía elaborada que realiza la comunidad intervenida, para ello se toman en consideración elementos de valoración como la efectividad de la información obtenida, problemas y limitaciones encontradas, resistencia, aceptación u oposición y grados de participación y compromiso. La comunidad analizará el resultado cartográfico haciéndose las siguientes preguntas:

a) ¿El ejercicio cubrió todos los temas esperados? Esta pregunta concierne la capacidad de la práctica en resolver y abordar todos los asuntos problemáticos propuestos en los procesos analíticos y su valoración estará determinada y ponderada en función de una resolución de aceptación o negación, desde el estar muy de acuerdo, con una puntuación de 5, hasta el desacuerdo, con una puntuación de 1 (Tabla $\mathrm{N}^{\circ} 1$ )

b) ¿La información generada en el ejercicio será útil para la revalorización de nuestro territorio? Esta interrogante corresponde a la facultad que ha tenido el ejercicio cartográfico en arrojar resultados aprovechables en su cometida de mejorar las reinterpretaciones del territorio y constituirse como base y diagnóstico para la construcción de futuras propuestas de desarrollo comunitario-territorial; su valoración será idéntica a la primera pregunta.

c) ¿Qué grado de dificultad asignamos al ejercicio? Determina el nivel de complicación y obstáculos encontrados en el ejercicio cartográfico de acuerdo a exigencias psicológicas, intelectuales y físicas; su valoración estará resuelta de acuerdo a escalas graduales de dificultad, desde muy fácil (5), hasta muy difícil (1).

d) ¿Cómo nos sentimos con la experiencia? Esta interrogante considera la percepción que tienen los participantes de su estado emocional durante el desarrollo de la práctica; su valoración estará determinada por grados de bienestar, desde muy cómodo (5) hasta muy incómodo (1).

e) ¿El tiempo asignado para el ejercicio fue el correcto? Esta pregunta constituye un elemento fundamental a la hora de analizar y establecer próximas estrategias de intervención. A pesar de que el tiempo fijado hubiera contemplado todos los temas esperados, no significa que hubiera sido el suficiente para un adecuado intercambio de ideas, creencias, sentires y vivencias, narrativas que dentro de un diálogo de saberes le dan sentido a la cartografía social. La valoración de esta pregunta irá de suficiente (5) hasta insuficiente (1).

Tabla $\mathrm{N}^{\circ} 1$ : valoraciones de las preguntas

\begin{tabular}{|l|l|l|l|l|}
\hline & \multicolumn{4}{|c|}{ Valoración del ejercicio cartográfico } \\
\hline Pregunta & 5 & 4 & 2 & 1 \\
\hline a) y b) & Muy de acuerdo & De acuerdo & Poco de acuerdo & Desacuerdo \\
\hline c) & Muy fácil & Fácil & Difícil & Muy difícil \\
\hline d) & Muy Cómodo/a & Cómodo/a & Incomodo/a & Muy incómodo/a \\
\hline e) & Suficiente & $\begin{array}{l}\text { Relativamente } \\
\text { suficiente }\end{array}$ & $\begin{array}{l}\text { Relativamente } \\
\text { insuficiente }\end{array}$ & Insuficiente \\
\hline
\end{tabular}

Fuente: Elaboración Propia 


\section{Resultados}

Los resultados obtenidos se describen a continuación a partir de las fases de IAP y se centran en el análisis de la cartografía del pasado y del presente, así como de su comparación.

\section{Fase 1. Hallazgo}

En la primera fase se realizaron entrevistas y reuniones (diciembre de 2017) con personal técnico del área de turismo del Gobierno Autónomo Descentralizado Provincial de Orellana (GADPO), de la Jefatura del Parque Nacional Yasuní y con personal especializado de la Dirección Provincial del Ministerio de Agricultura y Ganadería de Orellana (MAG-ORELLANA) con el objetivo de obtener información general del territorio y planificar la intervención. De este modo se seleccionaron las comunidades de Chiro Isla, Llanchama y Alta Florencia como áreas de intervención considerando aspectos geográficos, culturales y turísticos de acuerdo a las potencialidades de los paisajes de la zona. En este sentido, se identificaron las principales problemáticas y se obtuvo toda la información disponible de carácter documental y cartográfico. Cabe mencionar como aspecto positivo la disposición a colaborar en el proyecto de parte de las diferentes administraciones.

\section{Fase 2. Contacto inicial}

Durante el contacto inicial, (febrero de 2018) se efectuaron reuniones con agentes y líderes de las tres comunidades. El objetivo era cuádruple, en primer lugar, conocer de primera mano las problemáticas presentes en el territorio; en segundo lugar, presentar el proyecto de cartografía participativa; en tercer lugar, acordar la planificación de las actividades a realizar y, por último, diseñar estrategias que garantizaran la participación y empoderamiento de los miembros de las comunidades durante el ejercicio cartográfico.

\section{Fase 3. Intervención}

Durante la fase de intervención se obtuvo información tanto del pasado como del presente, sobre los límites y la zonificación, los asentamientos humanos, la actividad turística, los recursos naturales, las actividades extractivas y la cultura. Estos temas serán desarrollados en este apartado.

Uno de los aspectos interesantes que ofrece la cartografía participativa es la demarcación de fronteras para gestionar los derechos colectivos sobre el territorio. Tener unas fronteras reconocidas ofrece seguridad y protección y, además de permitir el reconocimiento de los derechos de la comunidad sobre el territorio, otorga cierta autonomía y apoya el mantenimiento de sus costumbres ancestrales. Bjorn Sietto (2009, citado en Camacho, 2017) expone la necesidad que tienen actualmente los pueblos indígenas de construir fronteras debido a las presiones que la administración pública ejerce sobre el territorio, como por ejemplo, la creación de nuevas unidades de ordenación con respecto a las figuras de protección de espacios naturales, o por la regulación de los usos del suelo y de los recursos naturales mediante la zonificación de actividades. Otra característica relacionada con las fronteras de las comunidades indígenas es que tradicionalmente estas no son concebidas como líneas fijas e invariables en el sentido occidental, sino como zonas o superficies porosas que eran acordadas y que fluctuaban dependiendo de las condiciones geográficas y sociales. La cartografía participativa sirve para mostrarle a la sociedad mayoritaria donde se encuentra el territorio que habita desde el tiempo de sus ancestros y exigir el reconocimiento de sus derechos (Camacho, 2017).

En relación a los límites y zonificación del pasado las tres comunidades comparten las mismas características; por una parte, las fronteras se configuraban indefiniblemente de acuerdo a condiciones y particularidades naturales, sociales, culturales y económicas y, por otra, no existía ningún tipo de división territorial o segregación del suelo de acuerdo a alguna función específica más allá de actividades dispersas como la pesca, los asentamientos humanos y la agricultura. En el caso de Chiro Isla, la parte oriental estaba delimitada por la comunidad Sinchichikta, la occidental por la comunidad Samona, al norte, el río Pacuya y al sur, el río Tiputini (Fig. N²:1a). Con lo que respecta a la Comunidad Llanchama en el norte aparece el río Tiputini, en el sur el río Jandia Yaku, en el este la comunidad Boca de Tiputini 
y en el oeste el Territorio Woarani ( Fig. $\mathrm{N}^{\circ} 2: 2 \mathrm{a}$ ). Para la comunidad Alta Florencia, de acuerdo a la Fig. $N^{\circ} 2: 3 a$, los límites estaban definidos al norte con el río Napo, al sur con el río Braga, al este con la comunidad Bello Horizonte y al oeste con la Hacienda Pando y la Comunidad Puerto Miranda.

En cuanto a los límites y zonificación del presente, Chiro Isla (Figura N²:1b) coincide con el del mapa del pasado, pero se incluye la Comunidad Playas de Cuyabeno al norte, el Parque Nacional Yasuní al sur, mientras que hacia el este y oeste se mantiene igual. La limitación en el presente para la Comunidad Llanchama, es fruto de un acuerdo con el pueblo Woarani y se incorpora la comunidad Puerto Quinche al norte, al sur el río Garza Yaku y el Parque Nacional Yasuní, al este se mantiene igual y oeste con el territorio Woarani y parte del Parque Nacional Yasuní (Figura N²:2b) En cuanto a los límites actuales de la Comunidad Alta Florencia es de destacar que coinciden con la delimitación de hace tres décadas. Finalmente, si bien las tres comunidades conocen la propuesta de uso de suelo del Plan de Manejo Comunitario de 2015 presentada por del Ministerio del Ambiente del Ecuador, no están de acuerdo con la definición de actividades ni con el porcentaje de superficie asignado a cada tipo de uso.

Figura $\mathrm{N}^{\circ}$ 2: Cartografía participativa. 1 Comunidad de Chiro Isla, 2. Comunidad de Llanchama, 3, Comunidad de Alta Florencia. Donde "a" se refiere al pasado (1980-1990) y "b" al presente (2018)
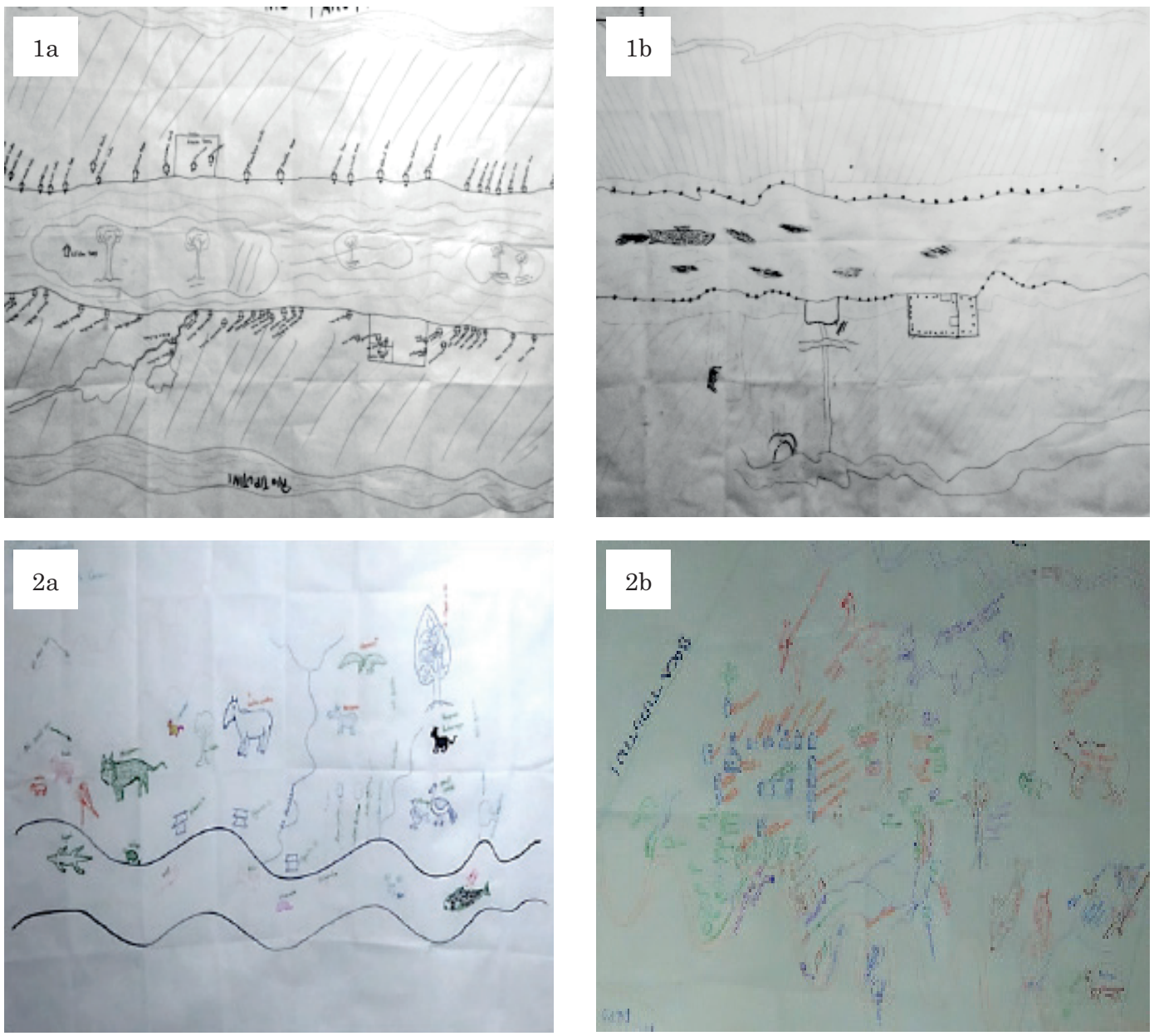

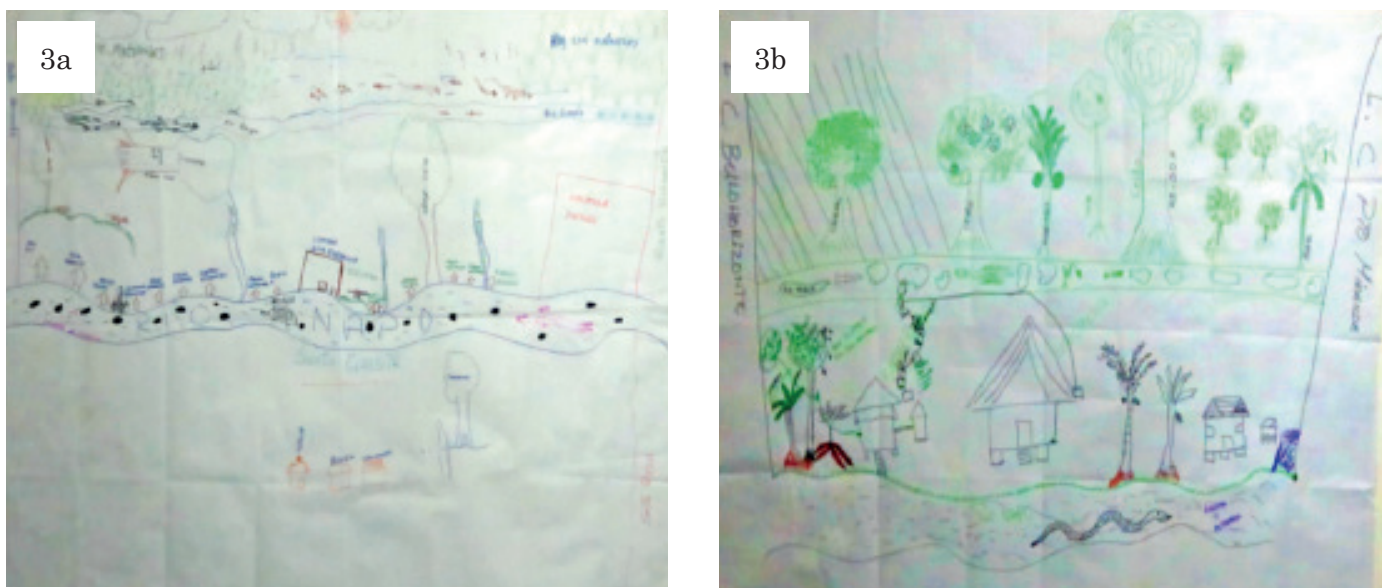

Fuente: Elaborado por los miembros de cada una de las comunidades.

De esta manera, la comparación de la delimitación del pasado y del presente permite afirmar que las comunidades en la actualidad perciben la necesidad de trazar fronteras estables y rígidas como elemento necesario para poder reclamar sus derechos, tanto fuera como dentro de la propia comunidad. También es interesante remarcar que los referentes utilizados como limitadores en el presente son más numerosos, incorporándose nuevas comunidades, pero sobre todo la figura del Parque Nacional Yasuní que es referente imprescindible en la identificación de las comunidades con respecto a su limitación territorial.

En lo que tiene que ver a los asentamientos humanos el ejercicio mostró una clara similitud en las tres comunidades respecto a los principales problemas presentes y sus causas: por un lado el aumento de la población colona debido a la falta de control y regulación en la oferta de terrenos baratos, por otro, la deficiencia de servicios básicos y el difícil acceso a la educación, consecuencia de la escasa inversión por parte de administración pública encargada.

En lo que concierne a los recursos naturales se revela en el presente la contaminación de los ríos y fuentes hídricas resultado de los derrames de petróleo, las descargas de contaminantes del transporte fluvial, y la generación de desechos por parte de un turismo irresponsable; así mismo se evidencia que en los últimos años, la caza indiscriminada por parte de personas que no pertenecen a la comunidad, la contaminación acústica generada por equipamientos para la actividad petrolera y la deforestación masiva ocasionada por acciones extractivas y el crecimiento del cultivo de palma, ha provocado la disminución de población de fauna y flora por lo menos en un 50\%. En la Figura 2 se puede distinguir claramente el número de ilustraciones de plantas y animales que hace 30 años existían y cohabitaban con las comunidades en comparación con las representaciones del presente en donde se muestra una pérdida considerable de especies de aves, reptiles, mamíferos y de flora extraordinaria.

En relación a la actividad turística, del análisis cartográfico se desprende que en el pasado no existían ninguna actividad relacionada a este sector, sin embargo, el turismo sostenible se venía pensando como actividad alternativa frente a la explotación petrolera. Así, en 2008 se crea la Red Solidaria de Turismo de la Ribera del Ría Napo que agrupaba a las comunidades con el objetivo de brindar servicios ecoturísticos a través de la revalorización de los paisajes ancestrales. No obstante, la falta de apoyo institucional, la poca regulación y control por parte del Ministerio del Ambiente, la exclusión de la participación comunitaria en la construcción de planes de manejo y ordenamiento del territorio y la intervención de operadoras de turismo extranjeras, han provocado que la Red de Turismo desaparezca y que los servicios ecoturísticos aislados que brindan las comunidades sean de baja calidad, lo que ha ocasionado una caída importante en la dinamización económica de estos sectores. Referente a las actividades extractivas el ejercicio muestra que hace 30 años, si bien se había iniciado las actividades de exploración y estudios de explotación petrolera, no se registraban impactos medioambientales. Con la concesión de grandes extensiones de territorio a empresas multinacionales se instala un oleoducto de crudos pesados y entre los años 2000 y 2005 se registraron importantes derrames de petróleo; en el presente la explotación continua y con ella los conflictos sociales y la degradación medio ambiental. 
Finalmente en lo que se refiere a la cultura, la importancia que han mantenido las comunidades por sus tradiciones es notable, a pesar de que ciertas prácticas como la shamaneria, el conocimiento curativo de las plantas, el sentido de sacralidad por los elementos y sus tradiciones orales prevalecen, los convencionalismo modernos relacionados a la migración mayormente de población joven y los sincretismos religiosos han difuminado ciertos contenidos trascendentes dentro de la preservación de la memoria histórica del territorio indígena.

\section{Fase V. Valoración}

De acuerdo a los criterios emitidos en el ejercicio cartográfico, la mayoría de los participantes de las tres comunidades (media del 77\%) coinciden que los contenidos propuestos en el ejercicio se desarrollaron en su totalidad, sin embargo, existieron algunos tópicos que surgieron a partir de la actividad dialógica que por falta de tiempo no se lograron profundizar (Tabla 2). En lo que se refiere a la utilidad de la información generada, en los tres casos de estudio el porcentaje se sitúa entre el 85 y el 90\%de manera que se puede afirmar que los participantes consideran que los datos obtenidos servirán de forma destacada para el reconocimiento, empoderamiento y mejor gestión de sus territorios. Desde su punto de vista permitirán exigir a la administración competente proyectos de conservación, fomento y preservación natural y cultural de sus comunidades. En cuanto a la dificultad que les significó realizar el ejercicio, más de la mitad de los asistentes (media del 70\%) en cada comunidad afirman que la actividad en términos generales fue sencilla, no obstante, para algunos comunitarios de edad avanzada, el diseño de mapas les supuso ciertas dificultades de interpretación pictográfica. Concerniente al nivel de bienestar, en las comunidades Chiro Isla y Llanchama, la totalidad de participantes aseguran haberse sentido a gusto y motivados en las actividades ejecutadas (100\%), empero, en el caso de Alta Florencia un $10 \%$ expresaba cierto grado de incomodidad debido a la falta de convocatoria realizada por parte del dirigente comunitario; aseveran que en este tipo de reuniones deberían haber sido convocados todos los miembros de la comunidad. Finalmente, en relación al tiempo asignado al ejercicio, un porcentaje mayor de participantes (40\%) en las tres comunidades manifiestan que el tiempo destinado (8 horas) para la práctica cartográfica fue insuficiente.

Tabla N²: Valoración del ejercicio cartográfico

\begin{tabular}{|c|c|c|c|c|c|c|c|c|c|c|c|c|c|c|c|c|}
\hline \multirow{2}{*}{$\begin{array}{l}\text { Elementos de } \\
\text { Valoración }\end{array}$} & \multicolumn{4}{|c|}{ Chiro Isla } & \multicolumn{4}{|c|}{ Llanchama } & \multicolumn{4}{|c|}{ Alta Florencia } & \multicolumn{4}{|c|}{ Media } \\
\hline & 5 & 4 & 2 & 1 & 5 & 4 & 2 & 1 & 5 & 4 & 2 & 1 & 5 & 4 & 2 & 1 \\
\hline $\begin{array}{l}\text { 1-Totalidad de los } \\
\text { temas cubiertos }\end{array}$ & 70 & 15 & 15 & 0 & 80 & 10 & 10 & 0 & 80 & 10 & 10 & 0 & 77 & 12 & 12 & 0 \\
\hline $\begin{array}{l}\text { 2-Información } \\
\text { generada útil para } \\
\text { el desarrollo del } \\
\text { territorio }\end{array}$ & 85 & 10 & 5 & 0 & 90 & 5 & 5 & 0 & 90 & 5 & 5 & 0 & 88 & 7 & 5 & 0 \\
\hline $\begin{array}{l}\text { 3-Dificultad } \\
\text { del Ejercicio } \\
\text { Cartográfico }\end{array}$ & 60 & 40 & 0 & 0 & 75 & 25 & 0 & 0 & 75 & 25 & 0 & 0 & 70 & 30 & 0 & 0 \\
\hline $\begin{array}{l}\text { 4-Percepción del } \\
\text { nivel de bienestar } \\
\text { en el desarrollo del } \\
\text { ejercicio }\end{array}$ & 90 & 5 & 5 & 0 & 100 & 0 & 0 & 0 & 100 & 0 & 0 & 0 & 97 & 2 & 2 & 0 \\
\hline $\begin{array}{l}\text { 5-Tiempo asignado } \\
\text { al ejercicio }\end{array}$ & 10 & 25 & 25 & 40 & 10 & 20 & 30 & 40 & 10 & 20 & 30 & 40 & 10 & 22 & 28 & 40 \\
\hline
\end{tabular}

Puntuación: 1 y 2. Muy de acuerdo (5), en desacuerdo (1). 3.- Muy fácil (5), muy difícil (1). 4.-Muy cómodo (5), muy incomodo (1). 5.- Suficiente (5), insuficiente (1)

Fuente: Elaboración Propia 


\section{Discusión de resultados y conclusiones}

Los resultados obtenidos en el presente estudio muestran la importancia de la aplicación de metodologías que favorecen una mayor participación de las comunidades en la diagnosis de sus territorios; en este sentido, la Investigación - Acción Participativa (IAP), un conjunto de procedimientos y herramientas, que, como menciona Santos (2008), considera la diversidad epistemológica de las realidades y la pluralidad de los saberes, ha posibilitado interpretar y caracterizar el objeto de estudio desde presupuestos intersubjetivos. La puesta en marcha de métodos participativos en el estudio ha permitido reafirmar la aproximación de Miguel Martínez (2009) que resalta que los sujetos investigados se convierten en auténticos coinvestigadores interviniendo activamente en el planteamiento del problema y convirtiéndose en programadores de su futuro. De acuerdo a este enunciado, es importante recalcar la apropiación por parte de los participantes del ejercicio y de los instrumentos metodológicos para reflexionar sobre el territorio y su realidad desde una visión endógena. Por lo mencionado, resulta evidente que metodologías alternativas como la IAP permiten llevar a cabo procesos innovadores de construcción del conocimiento a través de la creatividad, las relaciones y el diálogo. Además, muestra una relevancia conceptual y práctica en el diagnóstico de territorios indígenas, donde la comprensión de las formas de interpretación local del entorno es primordial en la caracterización del espacio-paisaje desde un distintivo geográfico, pero también desde el histórico, cultural y social. Esta notable característica (perceptiva), distinta a los mapas tradicionales en su metodología, estructura y contenido, ofrece una manera integral de entender los paisajes comunitarios.

La experiencia en las tres comunidades kichwas localizadas en la ribera del Río Napo de la Amazonía ecuatoriana esclarece las potencialidades de la cartografía social participativa con respecto a los estudios desarrollados hasta el momento. En el marco de los resultados vinculados al diagnóstico de los paisajes ancestrales de los territorios objetos de estudio, en la etapa del hallazgo a diferencia de otros estudios realizados en comunidades indígenas, no únicamente se contó con información de fuentes bibliográficas, sino que se trianguló información proveniente del personar directivo y técnico de gobiernos locales y responsables de la gestión del Parque Nacional Yasuní. Esta etapa del proyecto incorpora nueva información lo que incide en un grado de veracidad mayor con respecto a las consideraciones geográficas y de potencialidad al momento de diseñar estrategias de intervención. En lo que respecta al contacto inicial con las comunidades, la confianza generada a partir de las conversaciones con los líderes comunitarios constituyó un insumo fundamental a la hora de diseñar y evaluar la pertinencia y utilidad de las actividades dentro de la etapa de desarrollo. El interés demostrado por parte de los líderes comunitarios en participar en el ejercicio fue definitivo para la apropiación de la actividad del resto de comunitarios; estos resultados suponen semejanzas importantes en estudios de investigación con grupos poblacionales indígenas en los que se afirma que es fundamental conocer las dinámicas propias de cada grupo y coordinar con los representantes los contenidos y logística antes de proceder a la fase de ejecución del ejercicio (Velez et al, 2012). En la etapa de desarrollo o ejecución del taller se intentó representar cartográficamente los cambios que ha sufrido el paisaje de las tres comunidades debido a diferentes factores; en una línea de tiempo se representaron las características del territorio lo que posibilitó realizar análisis comparativos (De Sousa de Santos, 1991), tanto de la existencia de recursos naturales antes y después de las intervenciones extractivistas, como de la perdida de costumbres y significados de valor de patrimonio cultural. A través de los procesos dialógicos, el equipo de investigación intentó comprender los intereses colectivos lo que generó nuevas preguntas en relación a la planeación autónoma del territorio, la territorialidad construida dentro de lo cotidianeidad ancestral y la resignificación de los paisajes a través de la recuperación de la memoria histórica, elementos de significancia mayor para futuros estudios. En lo que concierne al proceso de valoración, el ejercicio no termina con la entrega del material cartográfico como muchos estudios lo proponen, sino con una evaluación por parte del participante de los contenidos desarrollados y de la metodología utilizada. Esta etapa es fundamental a la hora de moldear y legitimar estrategias de estudio poniendo al frente las necesidades y los intereses de los participantes. Finalmente, a pesar de que la cartografía participativa como metodología de análisis se ha difundido y ha aplicado a diferentes contextos, es sugerente considerar ciertos elementos diferenciadores cuando se trata de realizar estudios con comunidades indígenas en donde un acercamiento poco adecuado puede distorsionar, silenciar y sesgar la creatividad afectiva de los participantes (Carrión, et al. 2017).

Como conclusiones se puede apuntar que el paisaje dentro de un enfoque integral se presenta no solamente como algo corporal y concreto, sino que tiene características psíquicas y espirituales. La manifestación de esta relación, corporeidad-espiritualidad, concilia la transformación del territorio en 
un espacio vivo que se construye a través de las sensibilidades con las que las personas se relacionan con el paisaje. Estas consideraciones son fundamentales a la hora de establecer estrategias de diagnosis en lugares en donde el significado etno-territorial (orgánico) del paisaje permite comprender de manera transversal su transformación y deterioro. En este sentido, es imprescindible posicionar la necesidad de reconsiderar ontológicamente, epistemológicamente y metodológicamente la manera en cómo se analiza el paisaje y cómo se valora aboliendo enfoques que ubican al ser humano en una posición y relación de superioridad frente al territorio y a sus elementos.

La IAP como metodología de investigación busca trascender las costumbres pedagógicas con el propósito de proyectar al grupo poblacional de estudio como protagonista central de sus propias construcciones y valoraciones. Desde una perspectiva ideológica, la IAP se convierte en un instrumento de resistencia frente a imposiciones homogeneizadoras; desde el punto de vista epistemológico la IAP transgrede metodologías tradicionales en donde las relaciones de poder se manifiestan entre los investigadores y el grupo que va a ser investigado, este último relegado a un comportamiento y posicionamiento pasivo. Frente a esto, la IAP elimina los vínculos posicionales y convierte los participantes en sujetos activos con la capacidad de analizar, proponer y planificar sobre su propia realidad.

La cartografía social indígena (CSI) como una herramienta IAP se convierte en una estrategia fundamental de soberanía y en un instrumento político a la hora de tomar conciencia sobre la realidad de las comunidades tanto desde su interior como desde el exterior. La CSI no solo tiene como objetivo generar mapas sino resultados a través de los cuales se pueda prevenir y reducir los conflictos entorno al cuidado y gestión del paisaje y el territorio. En este sentido, la CSI procura ser parte de un registro testimonial que empodere y garantice la autodeterminación y soberanía sobre los propios proyectos de vida de las comunidades indígenas.

\section{Bibliografía}

Barrera, S. 2009. Reflexiones sobre Sistemas de Información Geográfica Participativos (sigp) ycartografía social. Revista Colombiana de Geografía. Volumen(18), 9-23. Disponible en file://C:/Users/pccarrion47/ Downloads/12798-33711-1-PB.pdf

Balcázar, F. 2003. Investigación acción participativa (iap): Aspectos conceptuales y dificultades de implementación. Fundamentos en Humanidades. Volumen (4),59-77. Disponible en https://www. redalyc.org/pdf/184/18400804.pdf

Becerra, T. 2016. Porque fracaso la Iniciativa Yasuni ITT. Instituto Universitario de Desarrollo y Cooperación IUDC- UCM. Madrid. Disponible en https://www.ucm.es/data/cont/docs/599-2016-02-04T36_Thaia_Becerra.pdf. Consultado 30 de marzo de 2018.

Carrión, P. et al. 2017. Cosmovisión indígena del paisaje: perspectiva sociocultural de preservación medioambiental. Revista Santiago. Volumen (145), 237-249. Disponible en https://go.gale.com/ps/ anonymous?id=GALE\%7CA536533111\&sid=googleScholar\&v=2.1\&it=r\&linkaccess=abs\&issn=00 $489115 \& \mathrm{p}=\mathrm{IFME} \& \mathrm{sw}=\mathrm{w}$

Colmenares, AM. 2012. Investigación-acción participativa: Una metodología integradora del conocimiento y la acción. Voces y Silencios: Revista Latinoamericana de Educación. Volumen (3), 102-115.

Santos, B.S. 2008. Reinventando la emancipación social. En Pensar el Estado y la sociedad: desafíos actuales. La Paz: CLACSO, Muela del Diablo Editores y Comuna.

De Sousa Santos, B. 1991. Una cartografía simbólica de las representaciones sociales Prolegómenos a una concepción posmoderna del derecho. Nueva Sociedad. Volumen (116), 18-38. Disponible en http://www.boaventuradesousasantos.pt/media/pdfs/Cartografia_simbolica_NuevaSociedad.PDF

Diez Temantine, J.M., Escudero, B., Carballeda, A., et al. 2012. Investigación e intervención desde las ciencias sociales, métodos y experiencias de aplicación. Versión en línea disponible en: https:// webcache.googleusercontent.com/search?q=cache:V7PVlrNLs8gJ:https://www.margen.org/Libro1. $\mathrm{pdf}+\& \mathrm{~cd}=1 \& \mathrm{hl}=\mathrm{es} \& \mathrm{ct}=\mathrm{clnk} \& \mathrm{gl}=\mathrm{ec}$. Consultado 28 de julio de 2019 .

Fals Borda y Rodríguez Brandao C. (1987) Investigación Participativa. Montevideo: La Banda Orienta

Fals Borda, O. 2008. Orígenes universales y retos actuales de la IAP (Investigación- Acción Participativa.

Peripecias, Versión en línea disponible en: http://www.peripecias.com/mundo/598FalsBordaOrigenesR etosIAP.html. Consultado 14 de agosto de 2019

Foucault, M. 1988. El sujeto y el poder. Revista Mexicana de Sociología. Volumen 50, 3-20.

FIDA. 2009. Buenas prácticas en cartografía participativa. Análisis preparado para el 
Fondo Internacional de Desarrollo Agrícola (FIDA). Versión en línea disponible en http://www.ifad.org/ pub/map/pm_web_s.pd. . Consultado 27 de noviembre de 2018

Furlong G.1936. Cartografía jesuítica del Río de la Plata (T. I y II). Buenos Aires: Talleres S. A., Casa Jacobo Peuser Ltda.

Guatarri, F. 2000. The Three Ecologies, Trans.I Pindar and P. Sutton. London and New Brunswick: Athlone Press.

Harley, B. 1989. Hacia una deconstruccion del mapa. La nueva naturaleza de los mapas. Cartographica. Volumen 26 (2), 1-20.

Kastrup, V. 2014. Cartografiar é traçar um plano común. Pistas do método da cartografia, 15-41

Kemmis, S. 1988. El currículum: va más allá de la teoría de la reproducción. Madrid: Morata

Lois, C. 2009. Imagen cartográfica e imaginarios geográficos. Los lugares y las formas de los mapas en nuestra cultura visual. SCRIPTA NOVA, Revista Electrónica de Geografía y Ciencias Sociales. Volumen (8), 298. Disponible en htt://www.ub.es/sn/sn-298.htm

Rocha, C.A. 2016. La investigación acción participativa. Una apuesta por la comunicación y la transformación social, Versión en línea disponible en http://www.ingebook.com/ib/NPcd/IB_BooksVis?cod_ primaria $=1000187 \&$ codigo_libro $=4475$. Consultado 18 de septiembre 2019

Martínez, M. 2009. Ciencia y arte en la metodología cualitativa. México: Trillas

Montoya, V. 2007. El mapa de lo invisible. silencios y gramática del poder en la cartografía. En Revista Universitas Humanística. Volumen (63), 155-179

Mora, F. 2011. LA Reserva de la Biosfera Yasuni y el turismo sostenible . La construcción del proyecto de la REST desde la interacción sociopolítica de actores. (Tesis de Maestría). FLACSO, Quito.

Sirvent, MT., Rigal, L. 2012. Investigación Acción Participativa: Un desafío en nuestros tiempos para la construcción de una sociedad democrática, Versión en línea disponible en: https://biblio.flacsoandes. edu.ec/catalog/resGet.php?resId=56482. Consultado 28 de julio de 2019

UN-HABITAT. 2010. State of the world's cities 2010-2011: Bridging the urban divide - Overview and key findings. Versión en línea disponible en: https://sustainabledevelopment.un.org/content/ documents/11143016_alt.pdf. Consultado 02 de febrero del 2019

Vélez, I. et al. 2012. Cartografía social como metodología participativa y colaborativa de investigación en el territorio afrodescendiente de la cuenca alta del río Cauca. Revista Colombia de Geografía. Volumen (21), 59-73. Disponible en: http://www.scielo.org.co/pdf/rcdg/v21n2/v21n2a05.pdf

Villaverde, X. et al. (2005). Parque Nacional y Reserva de Biosfera. Historia, problemas y perspectivas. Quito: Abya Yala

\section{Nota}

Este trabajo se enmarca en un proyecto de cooperación de la Fundación URV Solidària, del proyecto de investigación «El paisaje como valor colectivo. Análisis de su significado, usos y percepción social» (CHORA), financiado por el Ministerio de Ciencia, Innovación y Universidades (Programa Estatal de Fomento de la Investigación Científica y Técnica de Excelencia, Subprograma Estatal de Generación del Conocimiento. 2018-2020. CSO2017-82411-P), AEI/FEDER, UE y el Departament de Recerca i Universitats de la Generalitat de Catalunya (2017SGR22).

\section{Notas}

1 Desde 1998 se reconoce la zona intangible, como territorio designado a los pueblos en aislamiento voluntario.

Recibido:

Reenviado:

Aceptado:

Someticlo a
$07 / 07 / 2020$

$03 / 08 / 2020$

$23 / 11 / 2020$

Sometido a evaluación por pares anónimos 